\title{
Diffusive shock acceleration to relativistic energies in the solar corona
}

\author{
A. Sandroos ${ }^{1,2}$ and R. Vainio ${ }^{1}$ \\ 1 University of Helsinki, Department of Physics, Finland \\ e-mail: arto.sandroos@helsinki.fi \\ 2 Finnish Meteorological Institute, Helsinki, Finland \\ Received 2 September 2009 / Accepted 19 October 2009
}

\section{ABSTRACT}

\begin{abstract}
Aims. We study the effect of magnetic geometry on the efficiency of diffusive shock acceleration (DSA) of protons in the solar corona with emphasis on conditions that may lead to the formation of so-called ground level enhancements (GLEs) where the protons are accelerated into energies $\gtrsim 1 \mathrm{GeV}$.

Methods. We use Monte Carlo simulations of DSA in a semirealistic large scale coronal magnetic field near a bipolar active region. This active region is assumed to be the source region of a coronal mass ejection (CME) driving a shock wave in the corona. The shock geometry evolves in time, and the obliquity angle goes through a wide range of values from perpendicular to quasi-parallel. We consider the effect of the evolving magnetic geometry on the acceleration efficiency in five selected field lines.

Results. In most of the considered field lines the maximum proton energies are of the order of $100 \mathrm{MeV}$, which is rather typical for gradual solar energetic particle (SEP) events. We find that the DSA can be more effective on field lines where the shock starts out by being oblique and gradually turns quasi-perpendicular than on field lines where the shock starts perpendicularly.
\end{abstract}

Key words. acceleration of particles - shock waves - Sun: activity - Sun: coronal mass ejections (CMEs) - Sun: particle emission

\section{Introduction}

The most widely accepted acceleration mechanism of gradual SEP events is DSA (Axford et al. 1977; Bell 1978; Blandford \& Ostriker 1978; Krymskii 1977) in shock waves driven by CMEs. Gradual SEP events typically last for several days, have abundance ratios and charge states typical for the solar corona at $\lesssim 1 \mathrm{MeV}$ per nucleon energies and have power law energy spectra at low energies with a cut-off at higher energies. For protons, the cut-off is typically in the $10-100 \mathrm{MeV}$ range (see, e.g., Reames 1995).

At higher energies the gradual SEP events start to show substantial event-to-event variations (Tylka et al. 2005), especially in abundance ratios and charge states. It has been debated that in some cases these variations are caused either by a particle component accelerated at a solar flare accompanying the CME (Cane et al. 2006), or that the DSA accelerates a mixed seed particle population that contains remnant flare suprathermals in addition to the coronal material (Tylka et al. 2005). However, in the $1-10 \mathrm{MeV}$ nucl. ${ }^{-1}$ energy range, the event-to-event abundance variations are probably simply caused by variations in the cut-off energies that are due to shocks having different speeds etc.

The most extreme cases of gradual SEP events are the GLEs (see e.g. Reames 2009, and references therein), where secondary particles produced by $\gtrsim 1 \mathrm{GeV}$ protons in collisions with the particles in Earth's atmosphere are detected by ground-based neutron monitors. The GLEs are rather rare compared to gradual SEP events in general, with an occurrence rate of roughly one event per year vs. 20 events per year during solar maximum (see e.g. the event list in Cane et al. 2006), are usually associated with X-class solar flares in the western hemisphere, are associated with fast CMEs ( $\sim 1500 \mathrm{~km} \mathrm{~s}^{-1}$ median plane-of-sky speed), and are often accompanied with enhanced levels of high energy $(>10 \mathrm{MeV}$ ) pre-event protons at $1 \mathrm{AU}$ (Cliver et al. 1982; Cliver 2006). These characteristics indicate that there are some special conditions that must be met for $\gtrsim 1 \mathrm{GeV}$ protons to be detectable near Earth.

In previous studies (Sandroos \& Vainio 2007, 2009) we have performed Monte Carlo simulations of heavy ion acceleration in coronal shocks that have an evolving magnetic geometry and demonstrated that the resulting selective injection of particles into DSA can explain the observed high energy variations in gradual SEP events. This selective acceleration scenario was suggested by Tylka et al. (2005) and Tylka \& Lee (2006).

Here we study the acceleration of the highest energy protons in a similar global coronal model to the one we used in the previous studies, giving special attention to the effect of local magnetic geometry on the acceleration efficiency. Our code is a test particle simulation, i.e. wave growth due to the accelerated protons themselves (e.g., Bell 1978; Lee 1983, 2005) is not taken into account, even though it can occur in the corona.

We have developed Monte Carlo simulation models that employ the effect of self-generated waves (see e.g. Vainio \& Laitinen 2007, 2008; Vainio et al. 2009), but their computational efficiency is still not sufficient to employ a global field geometry with a proper treatment of the evolving shock and its downstream region. Furthermore, based on the observed peak proton fluxes at $1 \mathrm{AU}$, Vainio (2003) concluded that wave growth may not be fast enough to be important for the acceleration of $\sim 1 \mathrm{GeV}$ protons, which are accelerated and promptly released in the low corona. Consequently the plasma turbulence necessary for DSA to operate is assumed to be created by the background plasma processes.

We model an active region with a bipolar region that is embedded in a radial, large scale coronal magnetic field. This 


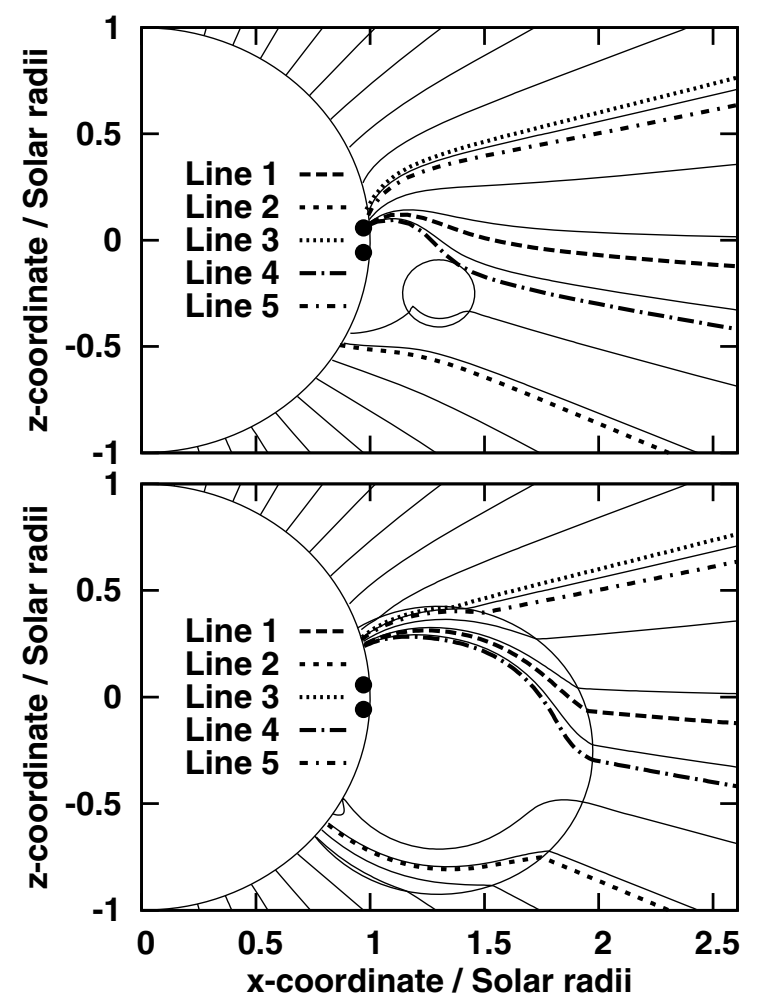

Fig. 1. (Top panel) Our coronal magnetic field model and the field lines used for particle injection. The circle depicts the shock front as it first intersects the injection field line 4 . The black dots show the positions of the monopoles that create the dipolar region (positive on top). (Bottom panel) The coronal field six minutes later than in top panel.

active region is assumed to be the source region of a CME driving a coronal shock wave. We study the injection and acceleration of protons on five selected magnetic field lines, each having a different shock obliquity angle profile, near this active region. We emphasize that we consider only the high energy tails of the proton spectra at energies $\gtrsim 100 \mathrm{MeV}$, as the spectra at lower energies could be dominated by the shock acceleration in the high corona and the solar wind, which we do not model. While our shock model is not completely realistic everywhere, we hope to produce the differences between different field line geometries qualitatively correct.

In Sect. 2 we briefly present our numerical model, in Sect. 3 show the results, discuss the results in Sect. 4, and give a summary of our results and discuss the conclusions in Sect. 5.

\section{Numerical model}

We model the shock as a spherical discontinuity with a constant radial expansion speed $V_{\mathrm{sh}}=1000 \mathrm{~km} \mathrm{~s}^{-1}$ and a constant gas compression ratio $X=3$. The center of the explosion is in the corona, which results in an evolving magnetic geometry of field lines swept by the shock (see Fig. 1). For injection field lines 1-4 the center of the explosion is at $\boldsymbol{R}_{\mathrm{sh}}=(1.3,0,-0.25) R_{\odot}$, and a value $\boldsymbol{R}_{\mathrm{sh}} \approx(1.357,0,-0.25) R_{\odot}$ was used for injection field line 5. The simulation code has been presented in detail in Sandroos \& Vainio (2009).

We employ a large Mach number approximation, i.e., assume that the shock expansion speed is clearly higher than the Alfvén speed in the upstream region. The upstream region is also assumed to be at rest. These approximations allow us to use simplified MHD jump conditions for oblique shocks, which are
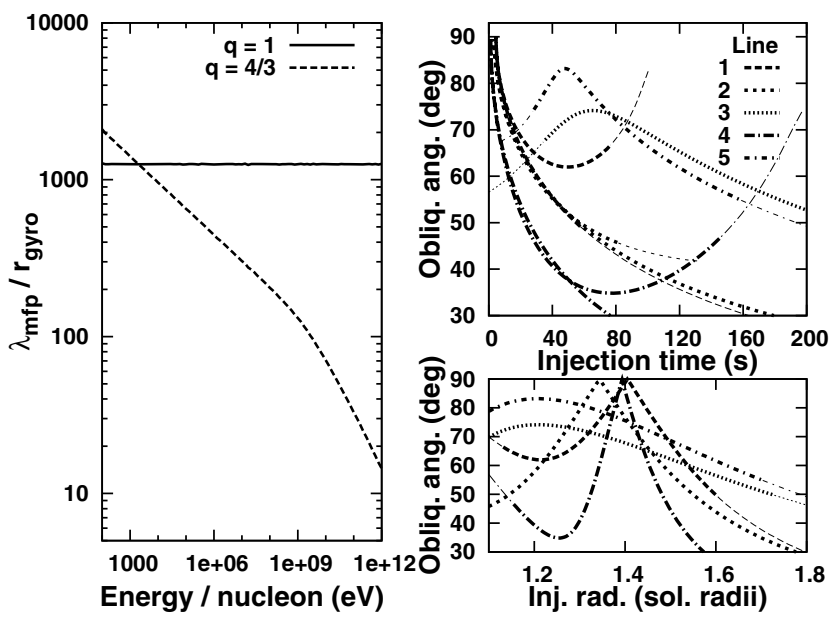

Fig. 2. (Left panel) The proton mean free paths relative to the gyro radii in our simulation model using values $q=1$ and $q=4 / 3$ for the Alfvén wave power spectrum. (Top right panel) Shock obliquity angle vs. injection time for the injection field lines 1-5. The curves have been shifted in time for clarity. Note that the parts of the field lines situated between the initial contact point and the surface of the Sun are also included in the figure, which is why most field lines appear twice. The region where particles were injected is drawn as a bold curve. (Bottom right panel) Shock obliquity angle vs. injection radius (in solar radii).

used to calculate the downstream plasma speed and magnetic field.

In the downstream region the plasma flow is assumed to be radially outwards from the center of the explosion. The downstream magnetic field can then be analytically solved for an arbitrary upstream magnetic field using the induction equation, assuming that diffusive effects can be neglected. In the downstream region there is a convective electric field while the upstream region is at rest, so the convective electric field vanishes.

The particle trajectories are computed using the full Lorentz force whenever the particles are in the downstream region or near the shock in the upstream region. In the upstream region, above $r \geqslant 1.6 R_{\odot}$ from the center of the Sun and far away from the shock, we assume that the ambient magnetic field is radial (from the center of the Sun), and compute the particle trajectories analytically using guiding center approximation. This is done simply to reduce overall computation time.

When using the full Lorentz force to compute particle trajectories, we model the pitch angle scattering by first calculating the time of the next scattering, which is chosen to be consistent with the scattering rate obtained from the quasilinear theory, then by computing the trajectory until that time is reached, and finally by randomizing the particle's pitch angle cosine. We take the power spectrum of the magnetic fluctuations to be a power law in wave number, $P(k)=P_{0}\left|k_{0} / k\right|^{q}$. We concentrate on simulations where values $q=1$ and $q=4 / 3$ have been used. The left panel of Fig. 2 shows the mean free paths vs. energy for the two values of $q$ for our scattering model.

We inject $1 \mathrm{MeV}$ protons on field lines 1-5 directly in front of the shock in the upstream region. The protons were removed from the simulation on the following conditions: the radial coordinate reached a value $r \geqslant 20 R_{\odot}$, the particles' distance to the shock front exceeded $0.05 R_{\odot}$ in the downstream, the simulation time exceeded $1200 \mathrm{~s}$, or the particle got too near the surface of the Sun $\left(r \leqslant 1.1 R_{\odot}\right)$. The right panel of Fig. 2 shows the shock obliquity angle vs. injection time profiles for each of the field lines. 


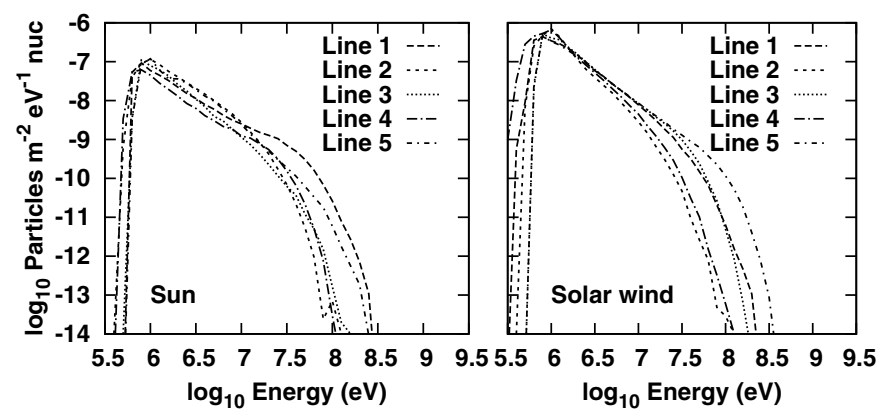

Fig. 3. The left panel shows the energy spectra from the $q=1$ simulation runs when only the protons hitting the surface of the Sun have been included (particles removed from simulation at radii $r_{\text {exit }} \leqslant 1.2 R_{\odot}$ ). The right panel shows the energy spectra for the same runs as in the left panel, but calculated from the protons escaping to the solar wind $\left(r_{\text {exit }} \geqslant 1.2 R_{\odot}\right)$.

\section{Results}

Figure 3 shows the energy spectra from the $q=1$ simulations, calculated from the particles hitting the surface of the Sun (left panel), and from those escaping to the solar wind (right panel). The particles were determined to "hit" the surface if their exit radius from the simulation satisfied $r_{\text {exit }} \leqslant 1.2 R_{\odot}$, while particles with $r_{\text {exit }} \geqslant 1.2 R_{\odot}$ were determined to escape to the solar wind.

The spectra resemble power laws with an exponential cutoff, although there are some deviations, especially in the surface spectra of lines 2 and 4 . Lines 1 and 5 are the most efficient accelerators to the surface, both producing $\sim 320 \mathrm{MeV}$ protons, with intensities larger by two orders of magnitude above above $100 \mathrm{MeV}$ than the rest of the injection lines. Line 5 is the most efficient accelerator to the solar wind, with lines 1 and 3 being the second best and about equal to each other.

Figure 4 shows the surface (left panel) and solar wind (right panel) energy spectra for the $q=4 / 3$ runs. The spectra are more power law-like than in the $q=1$ runs (Fig. 3 ). The line 1 is again an efficient accelerator towards the surface, but less so towards the solar wind. In these runs lines 3 and 5 are able to accelerate protons into the $\mathrm{GeV}$ energy range to the solar wind.

As illustrated in Figs. 3 and 4, the different shock obliquity profiles result in differences of roughly an order of magnitude in the cut-off energies between the different field lines. The intensities at a given energy above $\sim 100 \mathrm{MeV}$ can also vary by more than two orders of magnitude. The most notable injection lines are lines 3 and 5, which have a different obliquity profile from the other lines (Fig. 2). For these lines, the shock first intersects the lines close to the surface at an oblique angle, the shock obliquity then gradually increases and stays quasi-perpendicular for $\sim 50 \mathrm{~s}$ until finally starting to decrease as the shock expands (right panel of Fig. 2). The main difference between the lines 3 and 5 is that the maximum obliquity angle is larger for the injection line $5\left(\sim 83^{\circ}\right.$ vs. $\left.\sim 75^{\circ}\right)$, which allows faster acceleration during the quasi-perpendicular phase.

Figure 5 shows energy spectra from lines 1 and 5 in $q=4 / 3$ case with three different injection energies $(10 \mathrm{keV}, 100 \mathrm{keV}$, $1 \mathrm{MeV})$. The $1 \mathrm{MeV}$ spectra are the same as in the right panel of Fig. 3. The DSA is able to produce $\sim \mathrm{GeV}$ protons on line 5 even for $100 \mathrm{keV}$ injection energy, but the acceleration efficiency is drastically reduced somewhere between $10 \mathrm{keV}$ and $100 \mathrm{keV}$. Line 1 however has a much smoother dependency on the injection energy than line 5 .

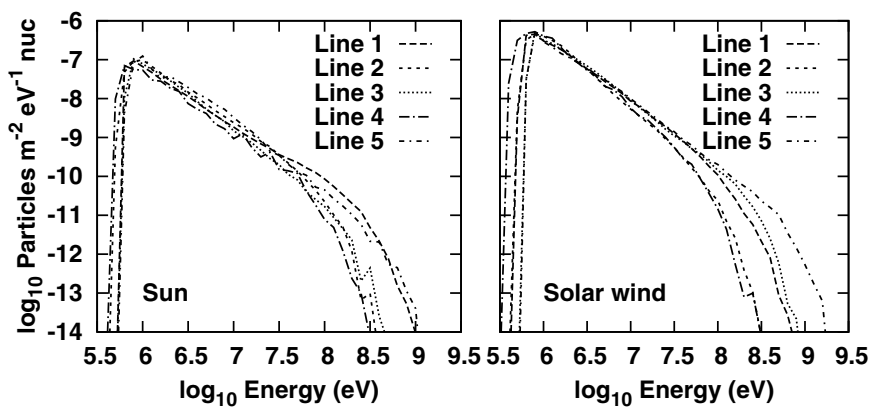

Fig. 4. Same as Fig. 3, but for $q=4 / 3$ simulation runs.

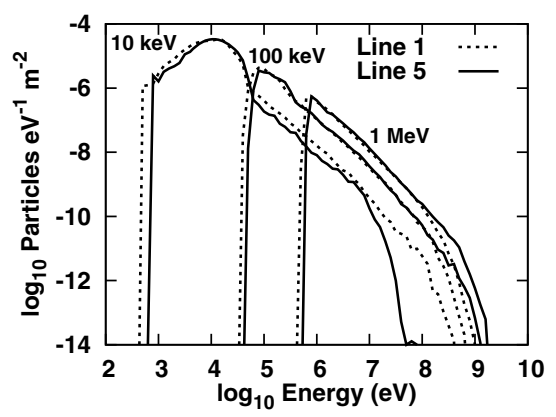

Fig. 5. A comparison of diffusive shock acceleration on injection lines 1 and 5, using three proton injection energies in $q=4 / 3$ runs.

\section{Discussion}

The higher energies obtained on line 5 are most likely due to the long-lasting quasi-perpendicular phase of shock evolution. The initial oblique phases on lines 3 and 5, before the shock obliquity angle peaks, also help by "pre-accelerating" the protons for the quasi-perpendicular phases. However, as explained below, these field lines are also somewhat more sensitive to the seed particle energies than the other field lines. Of course, if the level of turbulence is higher, field line meandering may enhance the injection (Giacalone 2005) by providing regions of less perpendicular geometry on all field lines. But this could also affect the efficiency of acceleration to the highest energies by increasing the particle mean free path in the shock-normal direction.

There is a significant difference on the injection efficiency between lines 1 and 2, which have very similar obliquity profiles, except for the parts of the field lines that are situated between the initial shock contact point and the surface of the Sun (Fig. 2). On line 1 there is a stronger magnetic mirror near the surface than on line 2, which accelerates the protons through adiabatic heating (Sandroos \& Vainio 2006). The field magnitude on line 1 is also about one order of magnitude larger than on line 2 (not shown here), which yields a higher scattering rate which in turn is probably responsible for the differences in the maximum energies.

At least $50 \%$ of the high energy protons accelerated on lines 1,2 , and 4 hit the surface of the Sun, as illustrated by the spectra shown in Fig. 4. This is caused by several factors. First, the stronger acceleration can be explained by the stronger scattering rates near the surface. In the case of lines 1 and 4, the shock also turns quasi-perpendicular for a second time near the surface after an oblique phase (see the right panel of Fig. 2), due to the field lines curving towards the bipole (Fig. 1). Additionally, it is difficult for the protons caught between the surface and the shock to escape to the solar wind due to the size of the downstream region between the surface and the $20 R_{\odot}$ exit 
boundary growing fast. On lines 3 and 5 this trapping between the surface and the shock does not occur.

Of course some of the protons that are trapped in our simulations might eventually be able to escape to the solar wind as the CME rises from the corona. However, our numerical model probably does not provide correct plasma behaviour near the surface, so it is unclear if the acceleration near the surface is as strong as in our simulations. Shocks near the surface should be affected by the boundary conditions at the Sun, which our model does not correctly take into account (e.g., Pomoell et al. 2008).

The drastic reduction of the acceleration efficiency on line 5 for lower injection energies is explained well by the injection threshold derived in Sandroos \& Vainio (2009),

$v_{\mathrm{thr}}=\frac{V_{\mathrm{sh}}}{\cos \psi_{\mathrm{us}}}\left(\frac{B_{\mathrm{us}}}{B_{\mathrm{ds}}}\right)^{1 / 2}=\frac{V_{\mathrm{sh}}}{\cos \psi_{\mathrm{us}}}\left(\frac{1+\tan ^{2} \psi_{\mathrm{us}}}{1+X^{2} \tan ^{2} \psi_{\mathrm{us}}}\right)^{1 / 4}$,

where $v_{\text {thr }}$ is the injection threshold velocity measured in the upstream rest frame, and $B_{\mathrm{us}}$ and $B_{\mathrm{ds}}$ are the magnitudes of the magnetic field in the upstream and downstream regions near the shock front respectively.

Using values appropriate to our simulations in Eq. (1), the corresponding injection threshold energy for the shock obliquity angle $\psi_{\text {us }}=65^{\circ}\left(85^{\circ}\right)$ is $E_{\text {thr }} \sim 11 \mathrm{keV}(\sim 230 \mathrm{keV})$. These are roughly the values of the obliquity angles for line 5 near the surface of the Sun and the maximum obliquity reached, correspondingly (see the right panel of Fig. 2). Thus, $10 \mathrm{keV}$ protons are injected only after the period of maximum shock obliquity and $100 \mathrm{keV}$ protons before and after it, while $1 \mathrm{MeV}$ protons are always injected. The particle acceleration on line 3 is similar to that on line 5, except that the injection threshold is somewhat lower during the quasi-perpendicular phase. On the other lines the acceleration starts when the injection threshold is exceeded for the first time and continues thereafter.

\section{Summary and conclusions}

We have presented results on Monte Carlo simulations of the DSA in the vicinity of an active region in the solar corona. The aim is to study how significent effect the field line geometry has on the maximum proton energies reachable within some minutes after a CME liftoff. The simulations model the lateral expansion of the CME as well as the evolution of the shock obliquity angle that follows.

The spectra calculated from our simulations are of the Ellison-Ramaty type (Ellison \& Ramaty 1985), i.e., power laws with an exponential cut-off at high energies. For most of the considered field lines the maximum proton energies are in the range of 100-300 MeV. We find that the magnetic geometry can have an effect of about one order of magnitude on the cut-off energies, and that on some special field geometries over $1 \mathrm{GeV}$ energies are possible, provided that seed particles with sufficiently high energies $(\gtrsim 100 \mathrm{keV})$ are available. Of course, other factors such as CME speed and the level of turbulence also contribute to the acceleration efficiency and its event-to-event variation. Note, however, that while faster CMEs accelerate particles more rapidly, they also require higher seed particle energies.

These results imply that GLEs are more easily created near regions where the coronal magnetic field is curved, e.g. in the vicinity of active regions or helmet streamers. These favourable regions may be quite small when compared to the area of the visible solar disk, implying that although $\sim \mathrm{GeV}$ protons may be created, most of the time an observer at $1 \mathrm{AU}$ is not magnetically connected to such a source region and thus these particles are not detected, in agreement with the low occurrence rate of GLEs.

The results presented here are consistent with some of the observed GLE events. Cliver (2006) found that for 15 out of 18 GLEs having elevated SEP backgrounds at 1 AU, the preGLE background was due to an eruption from the same active region that also produced the GLE. This is consistent with the observations that proton intensities in gradual SEP events are also more likely to be higher in events where a preceding CME has been launched from the same source region (Kahler 2001; Gopalswamy et al. 2004). In such events the preceding CME shock may provide both an enhanced turbulence level and energetic seed particle population for the subsequent CME (Li \& Zank 2005b).

We cannot exclude the possibility that some of the GLEs were created by the associated solar flare alone or by a CMEdriven shock reaccelerating the flare material. Observations in the $10-100 \mathrm{MeV}$ nucl. $^{-1}$ energy range suggest that flare material is present in most major SEP events (Cane et al. 2003). As pointed out by Li \& Zank (2005a), it may be possible to distinguish between different scenarios (DSA only, flare acceleration only, or DSA+flare), although the time-intensity profiles at high energies may be very similar in all scenarios.

In conclusion, particle acceleration at shocks near coronal active regions may produce $\mathrm{GeV}$ proton energies as long as the shock's strength, its speed, the level of ambient turbulence, seed particle populations and the magnetic geometry are all favourable for rapid DSA. This makes coronal shocks viable candidates for producing GLEs, at least in some of the events, although flare acceleration processes cannot be ruled out either.

Acknowledgements. The work of A.S. was funded by the Academy of Finland project No. 1110021.

\section{References}

Axford, W. I., Leer, E., \& Skadron, G. 1977, in Proc. 15th Int. Cosmic Ray Conf., 11,132

Bell, A. R. 1978, MNRAS, 182, 147

Blandford, R. D., \& Ostriker, J. P. 1978, ApJ, 221, L29

Cane, H. V., von Rosenvinge, T. T., Cohen, C. M. S., \& Mewaldt, R. A. 2003, Geophys. Res. Lett., 30, 5

Cane, H. V., Mewaldt, R. A., Cohen, C. M. S., \& von Rosenvinge, T. T. 2006, J. Geophys. Res., 111, 6

Cliver, E. W. 2006, ApJ, 639, 1206

Cliver, E. W., Kahler, S. W., Shea, M. A., \& Smart, D. F. 1982, ApJ, 260, 362

Ellison, D. C., \& Ramaty, R. 1985, ApJ, 298, 400

Giacalone, J. 2005, ApJ, 624, 765

Gopalswamy, N., Yashiro, S., Krucker, S., Stenborg, G., \& Howard, R. A. 2004, J. Geophys. Res., 109, 12105

Kahler, S. W. 2001, J. Geophys. Res., 106, 20947

Krymskii, G. F. 1977, Akademiia Nauk SSSR Doklady, 234, 1306

Lee, M. A. 1983, J. Geophys. Res., 88, 6109

Lee, M. A. 2005, ApJS, 158, 38

Li, G., \& Zank, G. P. 2005a, Geophys. Res. Lett., 32, 2101

Li, G., \& Zank, G. P. 2005b, in Proc. 29th Int. Cosmic Ray Conf., 1, 173

Pomoell, J., Vainio, R., \& Kissmann, R. 2008, Sol. Phys., 86

Reames, D. V. 1995, Adv. Space Res., 15, 41

Reames, D. V. 2009, ApJ, 693, 812

Sandroos, A., \& Vainio, R. 2006, A\&A, 455, 685

Sandroos, A., \& Vainio, R. 2007, ApJ, 662, L127

Sandroos, A., \& Vainio, R. 2009, ApJS, 181, 183

Tylka, A. J., Cohen, C. M. S., Dietrich, W. F., et al. 2005, ApJ, 625, 474

Tylka, A. J., \& Lee, M. A. 2006, ApJ, 646, 1319

Vainio, R. 2003, A\&A, 406, 735

Vainio, R., \& Laitinen, T. 2007, ApJ, 658, 622

Vainio, R., \& Laitinen, T. 2008, J. Atmosph. Solar-Terrestr. Phys., 70, 467

Vainio, R., Agueda, N., Laitinen, T., \& Battarbee, M. 2009, in Proc. 31st Int. Cosmic Ray Conf., in press 